\title{
LOCALISATION OF MINERALISED TISSUE IN A COMPLEX SPINNER FLASK ENVIRONMENT CORRELATES WITH PREDICTED WALL SHEAR STRESS LEVEL LOCALISATION
}

\author{
J. Melke ${ }^{1,2}$, F. Zhao ${ }^{1,2}$, B. van Rietbergen ${ }^{1}$, K. Ito ${ }^{1,2,3}$ and S. Hofmann ${ }^{1,2, *}$ \\ ${ }^{1}$ Orthopaedic Biomechanics, Department of Biomedical Engineering, Eindhoven University of Technology, \\ Eindhoven, the Netherlands \\ ${ }^{2}$ Institute for Complex Molecular Systems, Eindhoven University of Technology, Eindhoven, \\ the Netherlands \\ ${ }^{3}$ Department of Orthopaedics, UMC Utrecht, Utrecht, the Netherlands
}

\begin{abstract}
Spinner flask bioreactors have often been employed for bone tissue engineering. However, the reasons for their success in facilitating bone growth remain inconclusive. It was hypothesised that engineered bone tissue formation can be attributed to mechanical stimuli, which can be predicted in the tissue engineered construct. To test the hypothesis and draw conclusions as to how mechanical stimulation affects cell behaviour, a multidisciplinary approach using cell culture experiments and computational fluid dynamics (CFD) to simulate the complex flow within the spinner flask and scaffold was employed. Micro-computed tomography and histology showed that statically cultured human bone marrow derived stromal cells on silk fibroin scaffolds did not form extracellular matrix (ECM) or deposit minerals. However, constructs cultured at $60 \mathrm{rpm}$ resulted in ECM formation and mineralisation, mainly at the bottom of the scaffold (bottom: $78 \pm 7 \%$, middle: $17 \pm 5 \%$, top: $5 \pm 2 \%$ of total mineralised volume). Culturing at $300 \mathrm{rpm}$ led to a more homogeneously distributed ECM (bottom: $40 \pm 14 \%$, middle: $33 \pm 1 \%$, top: $27 \pm 14 \%$ of total mineralised volume). These observations were in agreement (Pearson correlation coefficient: $97 \%$ ) with the computational simulations that predicted maximal scaffold mineralisation, based on wall shear stress stimulation, in the bottom at $60 \mathrm{rpm}$ and in the main body at $300 \mathrm{rpm}$. Such combinations of CFD modelling and experimentation could advance our knowledge of the mechanical stimuli that cells experience in vitro and link them to biological responses.
\end{abstract}

Keywords: Bone tissue engineering, spinner flask bioreactor, computational fluid dynamics, scaffold.

*Address for correspondence: Dr Sandra Hofmann, Eindhoven University of Technology, PO Box 513, 5600 MB Eindhoven, the Netherlands

Telephone: +31 40-247 $3494 \quad$ Email: S.Hofmann.Boss@tue.nl

Copyright policy: This article is distributed in accordance with Creative Commons Attribution Licence (http://creativecommons.org/licenses/by-sa/4.0/).

\section{Introduction}

Tissue engineering and regenerative medicine are promising strategies for repairing or restoring diseased or fractured bones and for studying bone tissue development (O'Keefe and Mao, 2011; Tortelli et al., 2009). In vitro bone tissue engineering (BTE) approaches involve growing cells on either natural or synthetic biomaterials. Bone marrow derived mesenchymal stromal cells (MSCs) are capable of differentiating along the osteogenic lineage in the presence of various biochemical factors on different scaffold materials, such as silk fibroin and poly(lactic-co-glycolic acid) (PLGA), and to form bone-like tissue in vitro (Gentile et al., 2014; Melke et al., 2016). Even though there has been much progress, BTE approaches are far from clinical application. An understanding of fundamental processes of bone formation in vitro such as the (quantitative) link between mechanical forces, molecular biochemistry and gene expression is still lacking. Knowledge of ideal microenvironment properties is needed to translate in vitro findings to clinical applications and predict in vivo outcomes (Hulsart-Billstrom et al., 2016).

Due to the lack of vascularisation in vitro, reduced mass transport of nutrients and oxygen to and from the cells can affect cell migration and tissue ingrowth within the 3D scaffolds (Karageorgiou and Kaplan, 2005; Volkmer et al., 2008). To enhance mass transport in the culture system, bioreactors that can generate fluid flow within scaffolds are used (Alvarez-Barreto et al., 2007; Gaspar et al., 2012; Sikavitsas et al., 2002). The fluid flow within such a bioreactor can also 
generate fluid wall shear stress (WSS) on cells seeded within the scaffold pores (Zhao et al., 2015). In vivo, osteocytes react to WSS and translate mechanical stimuli into biochemical responses that affect bone remodelling (Klein-Nulend et al., 2012; Lanyon, 1993). In vitro studies demonstrate that osteoblasts are also sensitive to fluid-induced WSS. Fluid-induced WSS not only induces osteoblast proliferation and differentiation but also stimulates bone formation (Kapur et al., 2003; Norvell et al., 2004). WSS is capable of stimulating human MSCs (hMSCs) in vitro towards an osteoblastic phenotype in the absence of chemical induction (Yourek et al., 2010).

Among the different types of bioreactors, spinner flasks are often chosen due to their simplicity in utilisation. ALP activity and early osteogenic markers are significantly upregulated under dynamic conditions (30 rpm) in hMSCs, cultured for 3 weeks on PLGA scaffolds (Stiehler et al., 2009). Another longterm study with human dental pulp stem cells on silk fibroin scaffolds in spinner flask bioreactors shows an enhanced mineral deposition under dynamic conditions (120 rpm) as opposed to static culture after $47 \mathrm{~d}$. Specifically, $0.2 \pm 0.1$ bone volume fraction $\%$ (BV/TV\%) being measured under static conditions, while $4.9 \pm 1.6 \mathrm{BV} / \mathrm{TV} \%$ observed under dynamic conditions (Woloszyk et al., 2014).

Although simple in utilisation, the spinner flask bioreactor has a major disadvantage of the complex generated fluid flow within the bioreactor system and within the complex scaffold geometry. Therefore, the mechanical stimuli that cells receive within a scaffold are unknown. This makes it difficult to draw a conclusion on how mechanical stimuli modulate and ultimately affect cellular processes in vitro. Imitating mechanical environmental cues that cells experience in vivo, in a precise and controlled manner, could greatly improve and optimise cell and tissue responses in vitro (Lacroix et al., 2009). Controlling the mechanical environment within in vitro cell cultures would also lead to a greater reproducibility of experimental outcomes (Kirkpatrick et al., 2007; Martin et al., 2004).

In the current study, the working hypothesis was that the location of mineralised extracellular matrix (ECM), formed by cells within porous silk fibroin scaffolds, was dependent on and could be predicted by the mechanical stimulation applied to the cells in a spinner flask bioreactor. To test this, a multi-disciplinary approach was employed. According to previously defined mechano-regulation theories (Olivares et al., 2009; Vetsch et al., 2017) for mineralisation in vitro, the locations of mineralisation within the scaffold are predicted based on the flowinduced WSS in the scaffold pores. To compute the WSS, a computational fluid dynamics (CFD) model was developed for the spinner flask bioreactor system and an idealised scaffold geometry. Corresponding experiments were carried out using hMSCs seeded on silk fibroin scaffolds cultured in spinner flasks under both static and dynamic conditions.

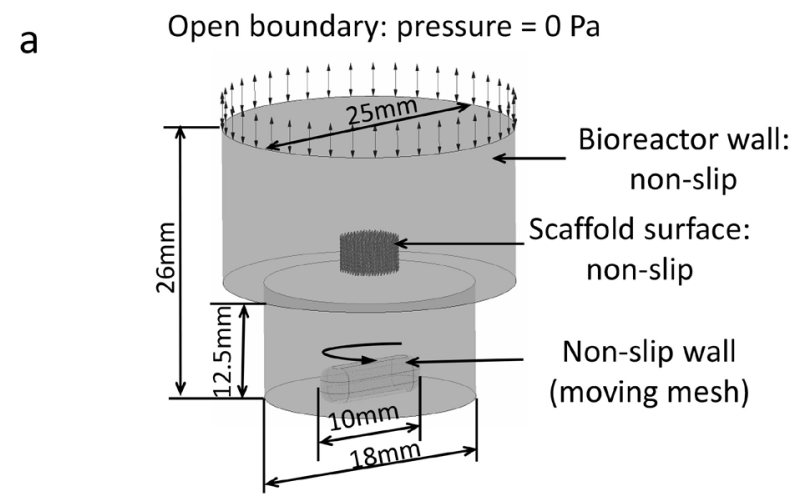

b

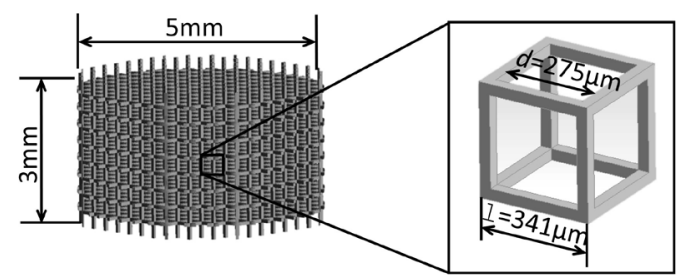

Fig. 1. Model parameters. (a) Boundary conditions of the computational fluid dynamics model based on the spinner flask bioreactor. (b) Dimensions of the entire scaffold and idealised geometry of the scaffold unit with a unit length of $341 \mu \mathrm{m}$ and pore size of $275 \mu \mathrm{m}$.

The experimentally obtained mineralised ECM distribution was correlated with the results of the CFD model. Such a model, capable of accurately determining the distribution of the (mineralised) ECM, could increase our understanding of which mechanical stimuli are sensed by cells and translated into biochemical reactions.

\section{Materials and Methods}

\section{Computational fluid dynamics model}

To quantify the mechanical stimulation generated within the scaffold and predict the area for osteogenesis and mineralisation, a CFD model was developed based on the spinner flask bioreactor (Fig. 1a). To save computational cost, a scaffold with idealised geometry, representing a regular 3D lattice structure which had a similar porosity $(\Phi)$ (Hofmann et al., 2007) and pore size (d) as the applied silk fibroin scaffold (i.e. $\Phi=90 \%$ and $d=275 \mu \mathrm{m}$ ), was used (Fig. 1b). A cubic porous unit was chosen based on the shape of $\mathrm{NaCl}$ crystals used as porogen for the silk fibroin scaffold. The density (Q) and dynamic viscosity $(\mu)$ of the culture medium were $1000 \mathrm{~kg} / \mathrm{m}^{3}$ and $1.45 \mathrm{mPa}$ 's, respectively (Olivares et al., 2009). To determine the flow type, the Reynold number $(R e)$ of the flow in the spinner flask bioreactor was calculated according to Hörmann et al. (2011):

$$
R e=\frac{n \cdot D^{2} \cdot \rho}{\mu}
$$


where, $n$ and $D$ were the stirrer rotational speed (rotations/s) and the length $(\mathrm{m})$ of the magnetic stir bar, respectively.

Two mechanical stimulation magnitudes were applied (60 and $300 \mathrm{rpm}$ ), which resulted in $R e$ numbers of 65 and 325, respectively, in the spinner region. The average Re number in the scaffold region, which is the region of interest in our study, was 31 and 125 for the 60 and $300 \mathrm{rpm}$ flow respectively. According to Hörmann et al. (2011), the flow in the scaffold region in the $60 \mathrm{rpm}$ case is laminar, while for the $300 \mathrm{rpm}$ case it is in the transition from laminar to turbulent. Menter (2009) successfully demonstrates the capability of a shear stress transport (SST) turbulence model to compute the drag force (shear force) at the boundary layers under transition flow. Therefore, a (SST) turbulence model was used for the transition flow.

The fluid domain was meshed with tetrahedron elements using a patch-conforming algorithm, which generated 654,800 tetrahedral elements. The surface mesh of the stirrer and scaffold were refined with element lengths of 10-400 $\mu \mathrm{m}$ and 10-150 $\mu \mathrm{m}$, respectively, which were controlled by the local curvature. The mesh was subdivided in curved regions until the individual elements spanned the angle between two neighbouring elements of 75-24 . To calculate the Re numbers in the scaffold region, the model was solved using the finite volume method (FVM) under steady state, using the commercial FVM codes (CFX, ANSYS Inc., US). Convergence was assumed when the root-mean-square of the residuals of the mass and the momentum was $<10^{-4}$. The fluid was considered Newtonian, such that the shear stress tensor ( $\tau i \mathrm{ij})$ on the scaffold surfaces $(\Gamma)$ could be calculated as:

$$
\tau_{i j}=\left.\mu \cdot\left(\frac{\partial v_{i}}{\partial x_{j}}+\frac{\partial v_{j}}{\partial x_{i}}\right)\right|_{x_{i} \in \Gamma}
$$

where, $v_{i}$ is the fluid velocity in the direction of $i$, $x_{j}$ is the $j$ th spatial coordinate and $\mu$ is the dynamic viscosity ( $\mu=1.45 \mathrm{mPa} \cdot \mathrm{s})$.

By applying previously reported WSS thresholds for osteogenesis (0.11-10 $\mathrm{mPa}$ ) and mineralisation of ECM (> $0.55 \mathrm{mPa}$ ) (Olivares et al., 2009; Vetsch et al., 2017), the area for osteogenesis and mineralisation, with low and high stimulation could be predicted from the output of the CFD model.

\section{Materials}

Dulbecco's modified Eagle medium (DMEM) and antibiotic/antimycotic (Anti-Anti) were obtained from Life Technologies (Bleiswijk, the Netherlands). Methanol was from Merck (Schiphol-Rijk, the Netherlands). Trypsin was from Lonza (Breda, the Netherlands). Foetal bovine serum (FBS) was from PAA laboratories (FBS Gold, Cölbe, Germany). Sodium chloride $(\mathrm{NaCl})$, hexafluoroisopropanol (HFIP), sodium carbonate $\left(\mathrm{Na}_{2} \mathrm{CO}_{3}\right)$, lithium bromide
(LiBr), dexamethasone, ascorbic acid-2-phosphate and $\beta$-glycerophosphate were obtained from Sigma Aldrich (Zwijndrecht, the Netherlands). Silkworm cocoons were purchased from Tajima Shoji Co., LTD. (Yokohama, Japan).

\section{Scaffolds}

Silk fibroin scaffolds were produced as previously described (Meinel et al., 2005; Nazarov et al., 2004). Briefly, Bombyx mori L. silkworm cocoons were degummed by boiling in $0.2 \mathrm{M} \mathrm{Na}_{2} \mathrm{CO}_{3}$ twice for $1 \mathrm{~h}$. Dried silk was dissolved in $9 \mathrm{M} \mathrm{LiBr}$ and dialysed against ultra-pure water (UPW) for $36 \mathrm{~h}$ using SnakeSkin Dialysis Tubing (molecular weight cut-off: 3.5 kDa; Thermo Fisher Scientific, Breda, the Netherlands). Dialysed silk solution was lyophilised (Freezone 2.5, Labconco, Kansas City, MO, USA) for $4 \mathrm{~d}$ and dissolved in HFIP, resulting in a $17 \%(\mathrm{w} / \mathrm{v})$ solution. $1 \mathrm{~mL}$ silk-HFIP solution was added to $2.5 \mathrm{~g}$ $\mathrm{NaCl}$ with a granule size between $250-300 \mu \mathrm{m}$ in a Teflon container and allowed to air dry for $4 \mathrm{~d}$. Silksalt blocks were immersed in $90 \%$ methanol in UPW for $30 \mathrm{~min}$ to induce $\beta$-sheet formation (Tsukada et al., 1994). $\mathrm{NaCl}$ was extracted in UPW for 2 d. Scaffolds were cut into disks of $3 \mathrm{~mm}$ height, punched with a $5 \mathrm{~mm}$ diameter biopsy punch and autoclaved in PBS at $121{ }^{\circ} \mathrm{C}$ for $20 \mathrm{~min}$.

\section{Cell culture}

hMSC isolation and characterisation from human bone marrow (Lonza, Walkersville, MD, USA) was performed as previously described (Hofmann et al., 2007). Pre-wetted scaffolds were seeded with 1 million cells each in $20 \mu \mathrm{L}$ control medium (DMEM, $10 \%$ FBS, $1 \%$ Anti-Anti) and incubated for $90 \mathrm{~min}$ at $37{ }^{\circ} \mathrm{C}$. The constructs $(n=4)$ were transferred to custom-made spinner flask bioreactors. Each bioreactor contained a magnetic stir bar and was placed on a magnetic stirrer (RTv5, IKA, Germany) in an incubator $\left(37^{\circ} \mathrm{C}, 5 \% \mathrm{CO}_{2}\right)$ except for media changes and the weekly imaging. Each bioreactor was filled with $5 \mathrm{~mL}$ osteogenic medium (control medium, $50 \mu \mathrm{g} / \mathrm{mL}$ L-ascorbic-acid-2-phosphate, $100 \mathrm{nM}$ dexamethasone, $10 \mathrm{mM} \beta$-glycerophosphate) and medium was changed 3 times a week for 4 weeks. To assess the cell location in the scaffold at the beginning of the culture, 4 constructs were sacrificed after the seeding process. The constructs were fixed for further analysis by a haematoxylin and eosin (H\&E) histological staining.

\section{Micro-computed tomography imaging $(\mu \mathrm{CT})$}

$\mu \mathrm{CT}$ measurements were performed on a $\mu \mathrm{CT} 100$ imaging system (Scanco Medical, Brüttisellen, Switzerland) after 4 weeks of culture. Scanning was performed at an isotropic nominal resolution of $17.2 \mu \mathrm{m}$, energy level was set to $45 \mathrm{kVp}$, intensity to $200 \mu \mathrm{A}, 300 \mathrm{~ms}$ integration time and two-fold frame averaging. A constrained Gaussian filter was applied to reduce part of the noise. Filter support was set to 1.0 and filter width sigma to 0.8 voxel. Filtered grayscale 
images were segmented at a global threshold of $22 \%$ of the maximal grayscale value. Unconnected objects smaller than 50 voxels were removed and neglected for further analysis. Quantitative morphometry was performed to assess mineralised ECM volume of the entire construct as well as the top, middle and bottom part of the construct using direct microstructural bone analysis as previously described for human bone biopsies (Hildebrand et al., 1999; van Lenthe et al., 2007).

\section{Histology}

Constructs were fixed in $10 \%$ neutral buffered formalin $\left(24 \mathrm{~h}\right.$ at $\left.4{ }^{\circ} \mathrm{C}\right)$, dehydrated in graded ethanol solutions and embedded in paraffin, bisected through the centre, cut into $6 \mu \mathrm{m}$ thick sections and mounted on poly-L-lysine coated microscope slides (Thermo Fisher Scientific, Breda, the Netherlands). Sections were stained with alizarin red to identify mineralisation, picrosirius red to identify collagen, alcian blue to identify glycosaminoglycans (GAGs) and H\&E for cell location. Figures show representative images of all the samples assessed.

\section{Statistical analysis}

Quantitative data are represented as mean \pm standard deviation $(n=4)$. $R$ version 3.3.3 was used for the evaluation of statistically significant differences. Under the assumption of normally distributed data, analysis of variance was performed followed by post hoc assessment using the Bonferroni method. Differences between groups were considered statistically significant at a level of $p<0.05$. Correlation of the average of the experimental data with the predicted value was calculated with the Pearson correlation coefficient.

\section{Results}

\section{Different spinner flask stirring rates resulted in distinct mechanical stimuli in porous scaffolds} The highest fluid velocity and WSS were predicted at the peripheral regions of the scaffold, particularly at the bottom of the scaffold at $300 \mathrm{rpm}$ (Fig. 2 and Fig. 3). The model predicted more mineralised ECM at 300 than at $60 \mathrm{rpm}$. At $60 \mathrm{rpm}$, mineralisation was predicted mainly at the very bottom of the scaffold ( $98.1 \%$ of total mineralised volume) and some peripheral regions. Middle and top part were predicted to have $1.6 \%$ and $0.4 \%$ of the total mineralised volume, respectively. Cells in the upper regions of the scaffold received stimuli that were too low to induce mineralised matrix deposition. At $300 \mathrm{rpm}, \mathrm{hMSC}$ in most of the scaffold volume received stimuli that triggered osteogenesis and induced mineralisation (bottom: $33.4 \%$, middle: $36.9 \%$, top: $29.6 \%$ of total mineralised volume). Too high and too low stimuli were only found at the very bottom and top part of the scaffold, respectively.

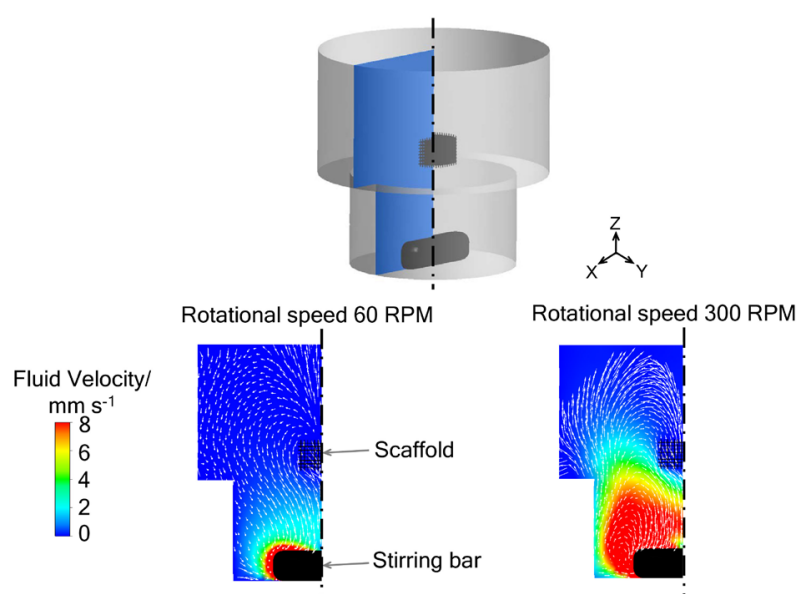

Fig 2. Fluid velocity distribution within the spinner flask bioreactor. Under (a) $60 \mathrm{rpm}$ and (b) $300 \mathrm{rpm}$, the vectors represent the flow path.

\section{Computational simulation predicted mineral formation}

Experimental formation of mineralised tissue was measured with $\mu \mathrm{CT}$ and visually compared with the prediction of mineralised ECM formation from the computational model (Fig. 4a). As expected, $\mu \mathrm{CT}$ images of the tissue-engineered constructs after 4 weeks showed that statically cultured constructs showed no mineralised volume $\left(0.0 \pm 0.0 \mathrm{~mm}^{3}\right)$ (Fig. 4b). Mineralised volume was only observed in constructs cultured dynamically at either 60 $\left(0.5 \pm 0.3 \mathrm{~mm}^{3}\right)$ or $300 \mathrm{rpm}\left(3.9 \pm 1.3 \mathrm{~mm}^{3}\right)$ (Fig. $\left.4 \mathbf{b}\right)$. As is typical for cell culture studies, a large variation was found within each group. A significant difference in mineralised volume was observed between the static group and the $300 \mathrm{rpm}$ group as well as the $60 \mathrm{rpm}$ group and the $300 \mathrm{rpm}$ group $(p<0.01)$. The volumes of the mineralised tissue in the bottom, middle and top part of the scaffold (Fig. 4c) were quantified for both the cell culture experiment (Fig. $4 \mathbf{d}, \mathbf{e})$ and the computational simulation (Fig. $4 \mathbf{f}$ ) to evaluate whether model and experimental results correlated. Constructs cultured at $60 \mathrm{rpm}$ developed $5.4 \pm 2.5 \%$ of the total mineralised volume in the top part and $16.5 \pm 5.2$ in the middle, which were both significantly less than the total mineralised volume in the bottom part $(78.1 \pm 14.3 \%)$ of the scaffold (Fig. $4 d$ ). Constructs cultured at $300 \mathrm{rpm}$ had $26.7 \pm 13.9 \%$ of the total mineralised volume in the top part of the scaffold, $32.8 \pm 0.7 \%$ in the middle and $40.5 \pm 14.3 \%$ in the bottom part of the scaffold (Fig. 4e). The averaged total mineralised volume fractions in bottom, middle and top regions of the scaffold were correlated with the predicted values and resulted in a Pearson correlation coefficient of $97 \%$.

\section{Computational simulation predicted mineralised extracellular matrix distribution}

Histological staining was performed to visualise both mineralised and non-mineralised ECM and how it was distributed in the scaffold. The results suggested 


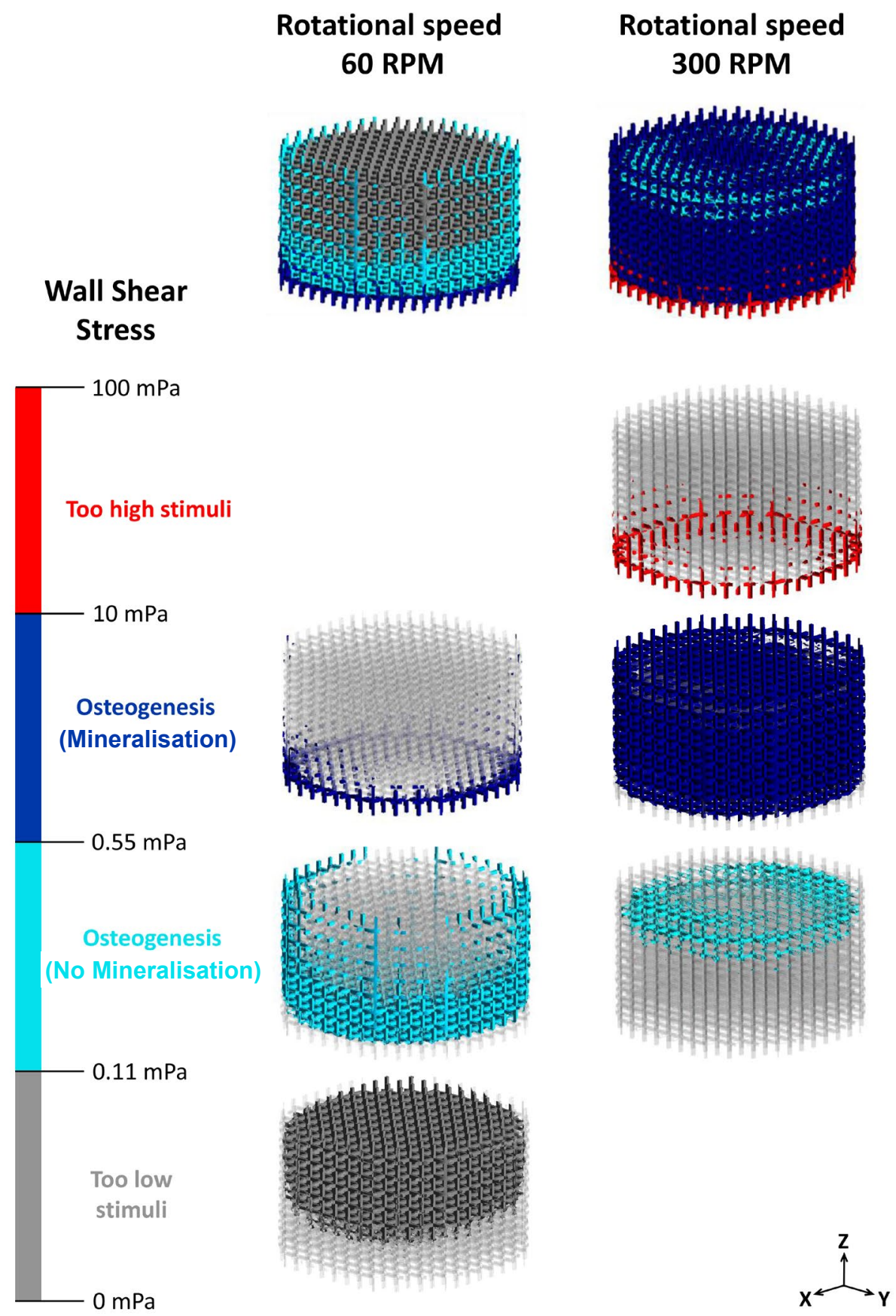

Fig. 3. Prediction of regions within the silk-fibroin scaffold receiving different mechanical stimuli within a spinner flask bioreactor. Bioreactor was set at a rotational speed of either 60 or $300 \mathrm{rpm}$. Corresponding wall shear stresses are grouped in too low/high stimuli that do not result in osteogenesis, stimuli that lead to osteogenesis without mineralisation and stimuli that result in osteogenesis with mineralisation of extracellular matrix.

that even though the cells were seeded on top of the scaffold, they either attached directly or migrated through the complete scaffold volume in all groups (Fig. 5). Statically cultured constructs showed a slight deposition of collagen and GAGs, and almost no mineralisation (Fig. $5 \mathbf{a}, \mathbf{b}, \mathbf{c}$ ). The borders of the construct showed an increased alignment of collagen and GAGs, as cells in these regions experienced a 2D surface rather than a 3D surface found in the scaffold. Despite the size of the constructs, which was much larger than the $200 \mu \mathrm{m}$ diffusion limit, there was no observable difference in matrix deposition between the centre of the scaffold and the border. Constructs cultured at $60 \mathrm{rpm}$ showed deposition of collagen and its mineralisation in the pores on the lower side of the scaffold (Fig. 5d,f). GAGs were mainly limited to the bottom part of the scaffold (Fig. 5e). Mineralisation was in agreement with the $\mu \mathrm{CT}$ data, which showed mineralisation mainly at the bottom of the scaffold at $60 \mathrm{rpm}$. At $300 \mathrm{rpm}$, regions with 
a

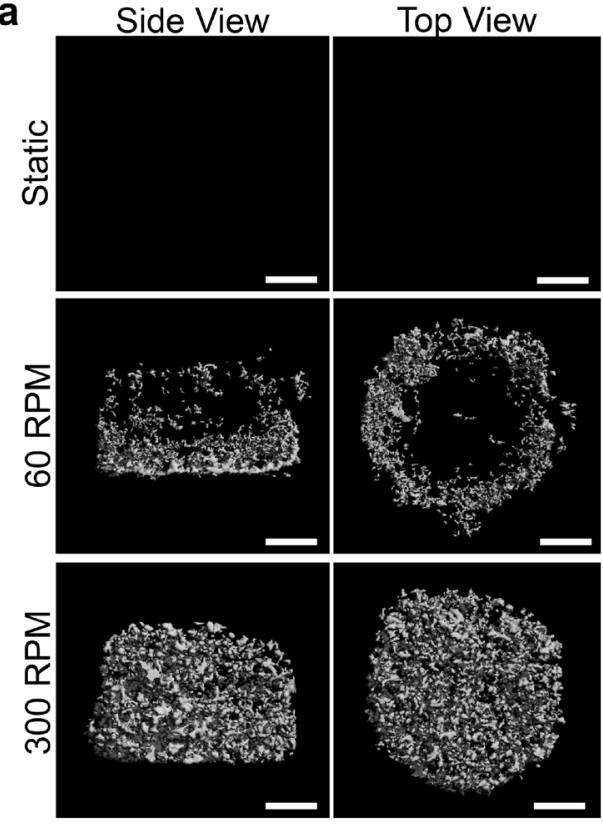

b

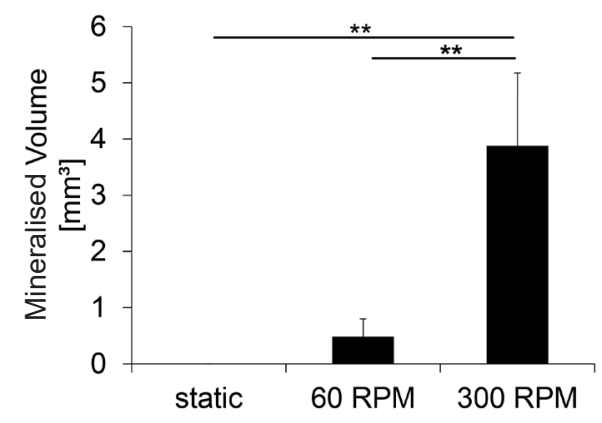

C

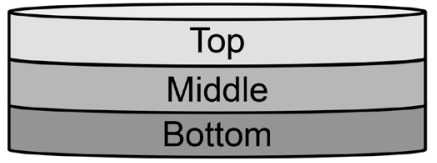

d

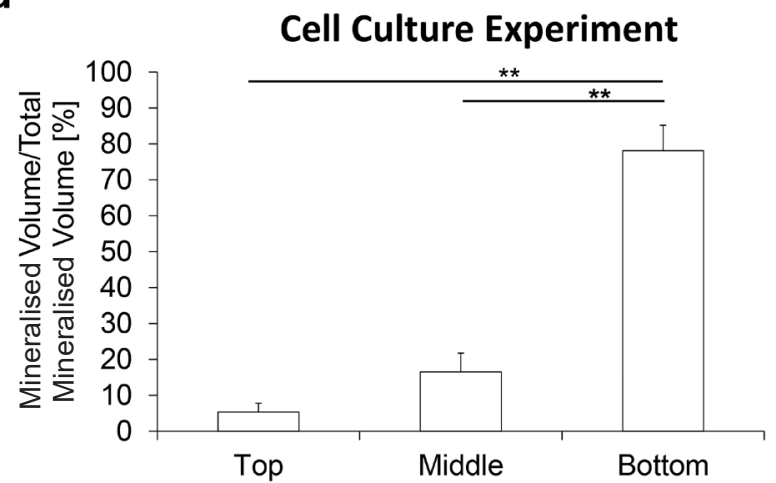

e

\section{Cell Culture Experiment}

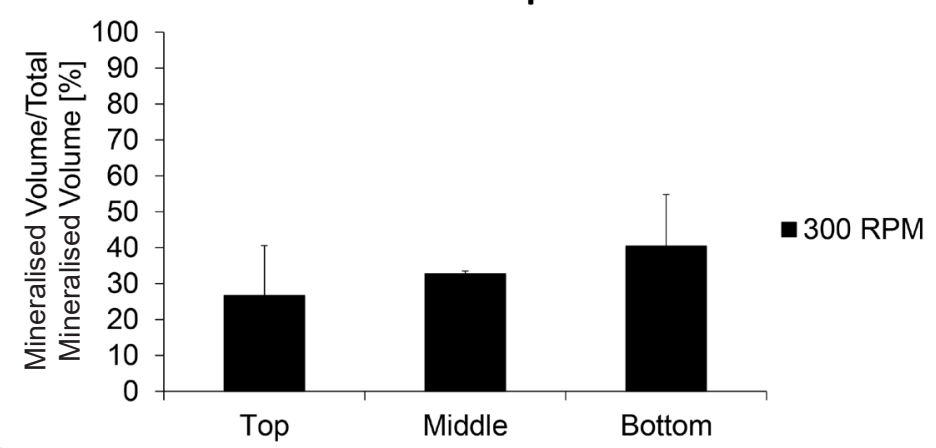

Computational Simulation

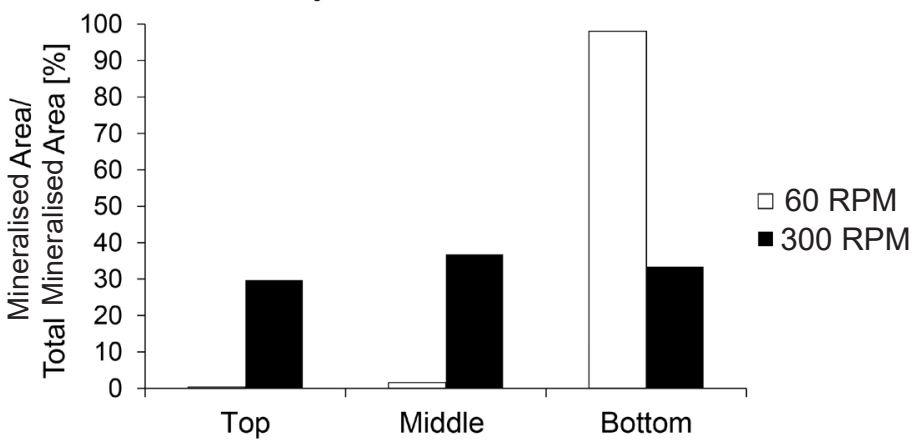

Fig. 4. 3D $\mu \mathrm{CT}$ images of human mesenchymal stromal cells on silk fibroin scaffolds. (a) Mineralisation after 4 weeks of culture under static or dynamic (at $60 \mathrm{rpm}$ or $300 \mathrm{rpm}$ ) culture in a spinner flask (a). The upper median sample of total mineralised volume (MV) of each group is shown in side view (left column) and side view (bottom row): Scale bar $=1 \mathrm{~mm}$. (b) MV per scaffold after 4 weeks of culture. Significantly more MV was produced in constructs cultured at $300 \mathrm{rpm}$ compared to constructs cultured at $60 \mathrm{rpm}$ and statically. (c) Schematic representation of the evaluated scaffold area. (d) MV/Total MV produced in the bottom, middle and top part of constructs cultured dynamically at $60 \mathrm{rpm}$. Significantly more MV/Total MV was produced in the bottom part than in the middle and top part of the constructs. (e) MV/Total MV produced in the bottom, middle and top part of constructs cultured dynamically with $300 \mathrm{rpm}$. No significant difference was found between top, middle and bottom part. (f) Mineralised Area/ Total Mineralised Area as calculated by the computational simulation. Error bars represent mean \pm standard deviation. ${ }^{* *} p \leq 0.01$.

high mineralisation, collagen and GAG deposition (Fig. 5g,h,i) were observed throughout the scaffold volume, with larger and more prominent structures in the centre of the scaffold. These findings were in agreement with the $\mu \mathrm{CT}$ results, which showed mineralisation throughout the whole scaffold volume at $300 \mathrm{rpm}$.

\section{Cells migrated through the scaffold during} culture

Histological staining was performed to visualise the cells present in the scaffold after seeding and after 4 weeks of static or dynamic culture. After seeding, hMSCs could be found predominantly in the top region of the scaffold (Fig. $6 \mathbf{a}, \mathbf{b}, \mathbf{c}$ ), which can be attributed to the static seeding technique. The cells 
Collagen
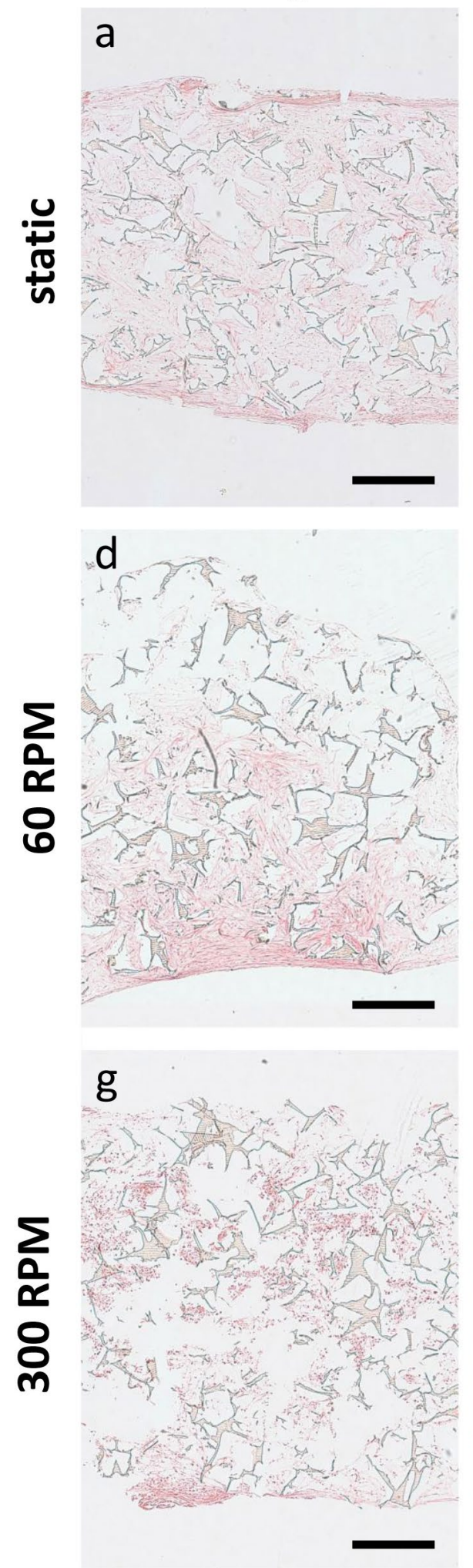

GAGs

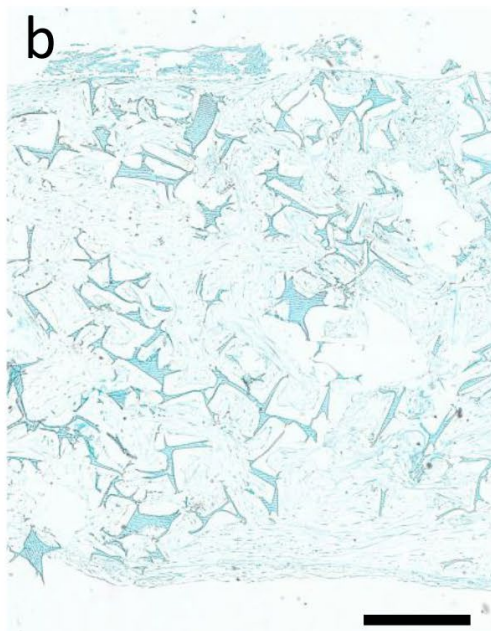

e

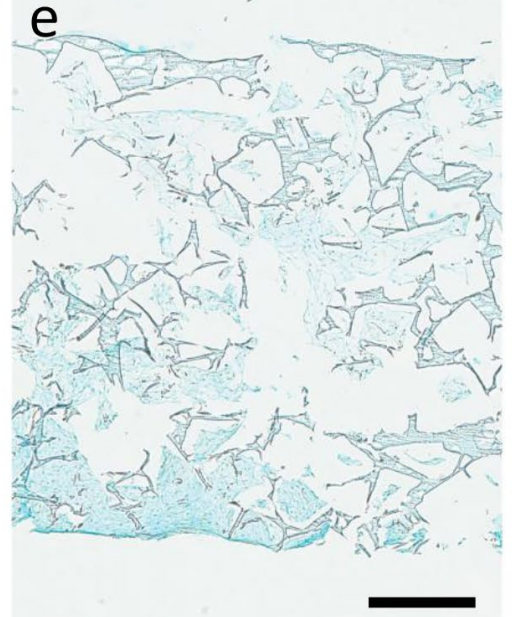

$\mathrm{h}$

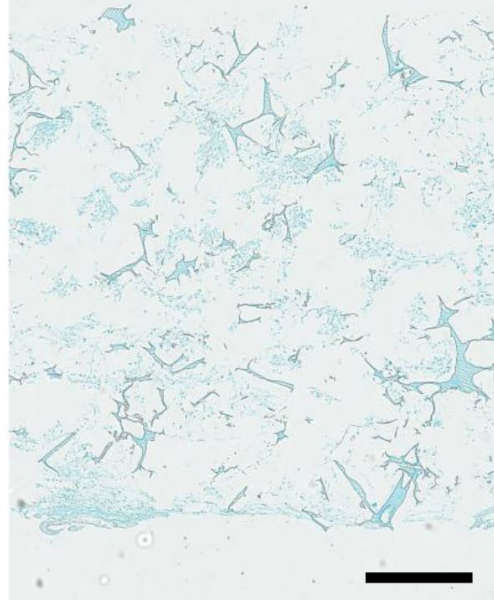

Mineralisation
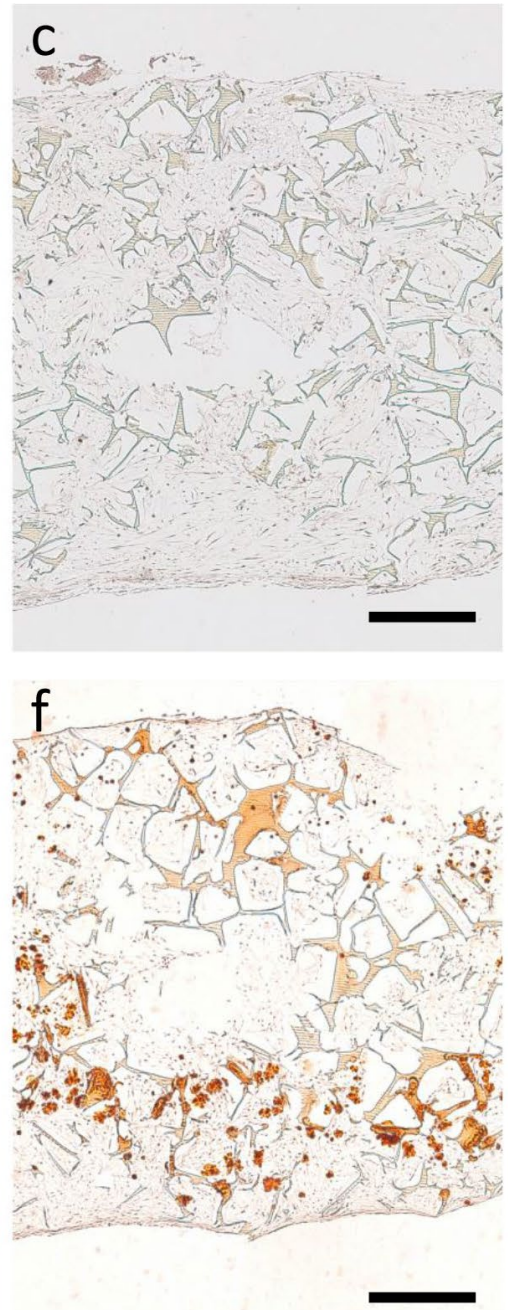

i

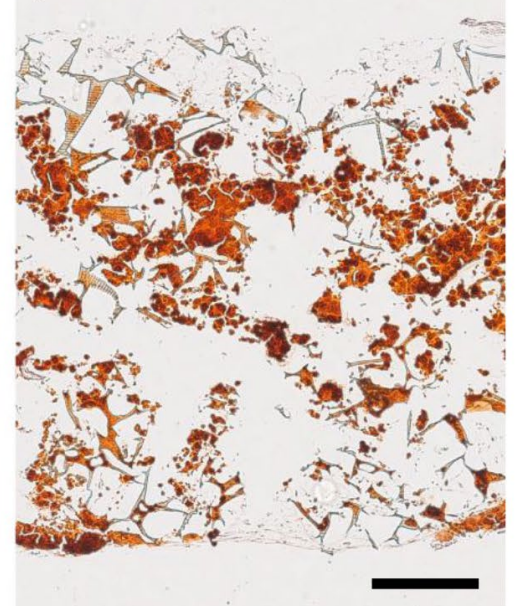

Fig. 5. Histological staining of human mesenchymal stromal cells on silk-fibroin scaffolds. Cultured statically $(\mathbf{a}, \mathbf{b}, \mathbf{c})$ or dynamically at either $60 \mathrm{rpm}(\mathbf{d}, \mathbf{e}, \mathbf{f})$ or $300 \mathrm{rpm}(\mathbf{g}, \mathbf{h}, \mathbf{i})$ for 4 weeks in spinner flask bioreactors. Histological sections were stained for collagen (picrosirius red; $\mathbf{a}, \mathbf{d}, \mathbf{g}$ ), GAGs (alcian blue; $\mathbf{b}, \mathbf{e}, \mathbf{h}$ ) and minerals (alizarin red; $\mathbf{c}, \mathbf{f}, \mathbf{i}$ ). The upper side of the scaffold is on the top of the picture. Low levels of collagen and GAGs were formed under static conditions. Dynamic conditions at $60 \mathrm{rpm}$ and $300 \mathrm{rpm}$ lead to collagen and GAG deposition at the bottom or throughout the scaffold, respectively. While no mineralisation occurred in the static group, mineral deposition was visible at $60 \mathrm{rpm}$ and $300 \mathrm{rpm}$ at the bottom or throughout the whole scaffold, respectively. Scale bar: $500 \mu \mathrm{m}$. 

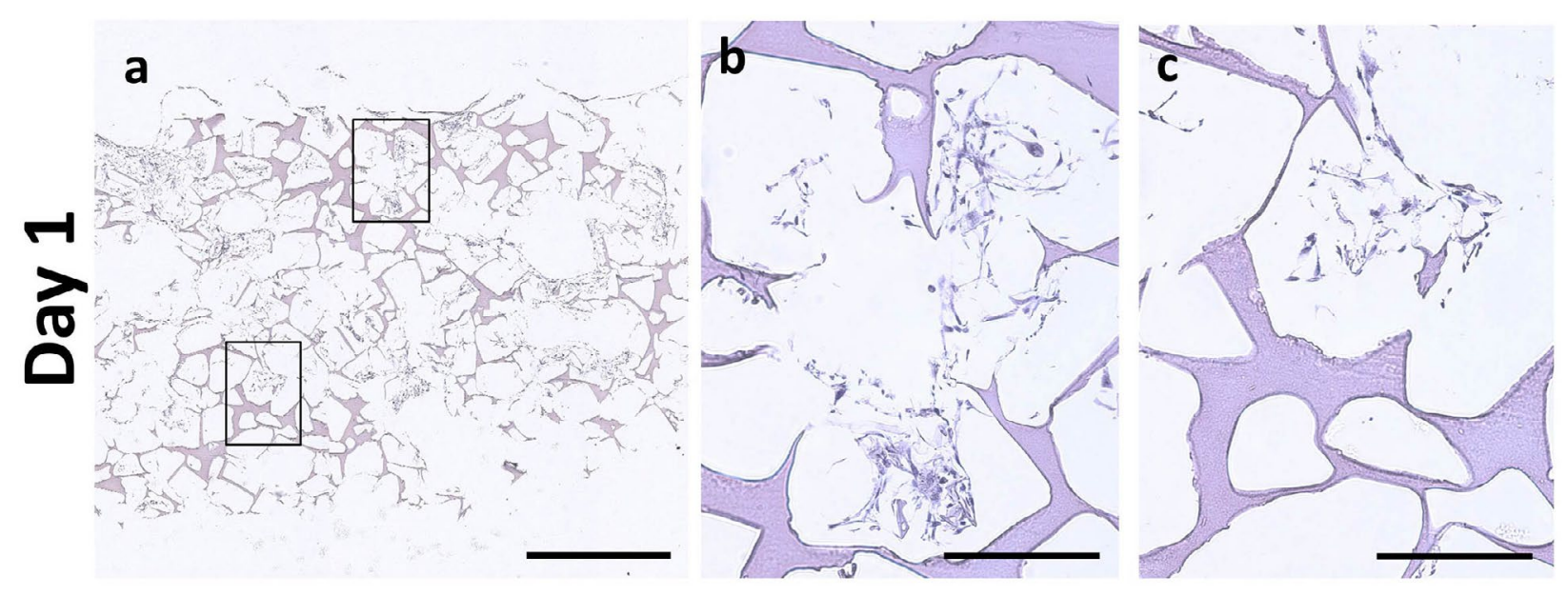

static

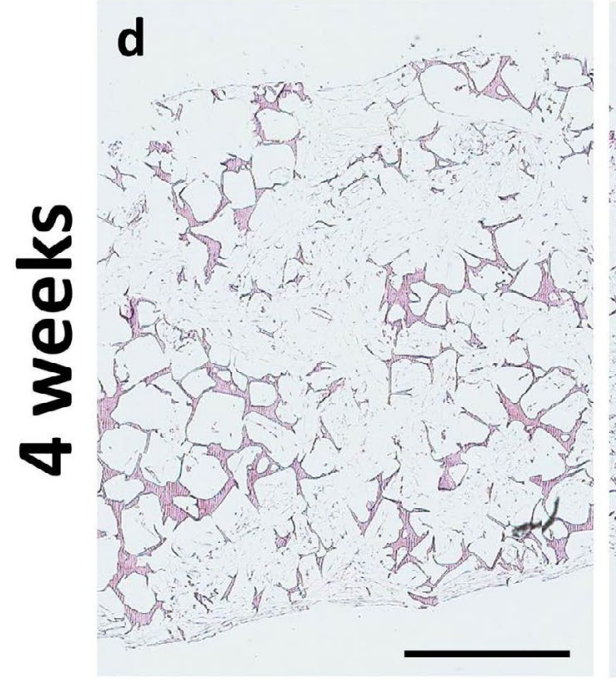

60 RPM

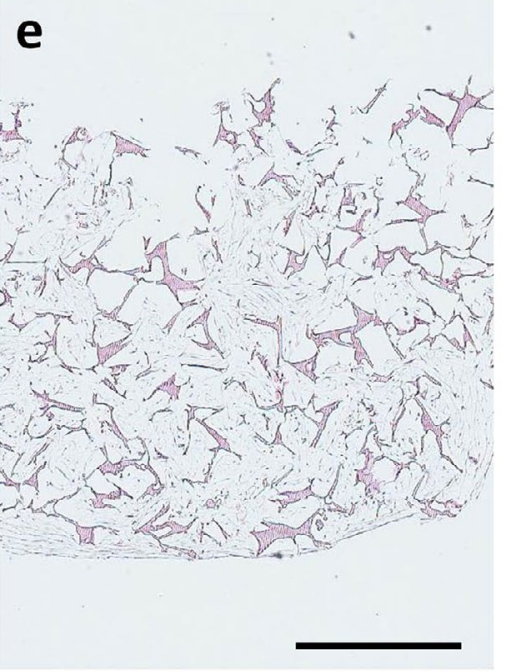

300 RPM

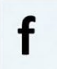

f

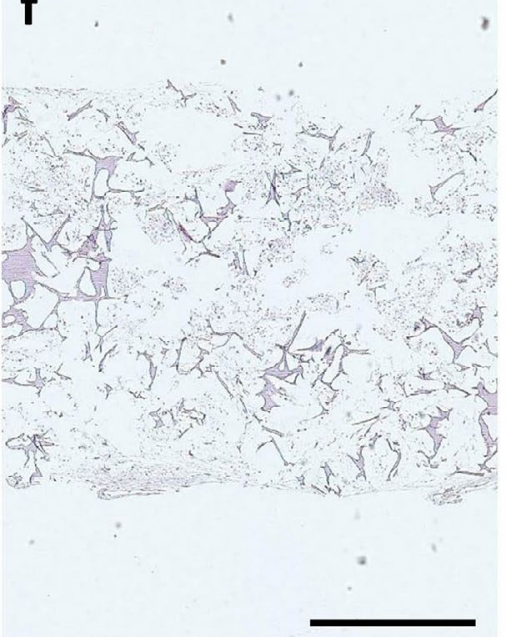

Fig. 6. H\&E staining of silk-fibroin scaffolds seeded with human mesenchymal stromal cells. Constructs after being seeded $(\mathbf{a}, \mathbf{b}, \mathbf{c})$ and after 4 weeks in osteogenic medium under static $(\mathbf{d})$ or dynamic cultures $(\mathbf{e}, \mathbf{f})$. Cells could be found mainly in the top part of the scaffold after seeding. During the 4-week culture the cells migrated throughout the whole scaffold volume. Scale bars: $1000 \mu \mathrm{m}(\mathbf{a}, \mathbf{d}, \mathbf{e}, \mathbf{f})$ and $200 \mu \mathrm{m}(\mathbf{b}, \mathbf{c})$.

formed clusters inside the scaffold pores and formed few attachment points to the scaffold structure. After 4 weeks of culture, cells could be found attached to the scaffold structure throughout the constructs (Fig. 6d,e,f). These results suggested that after being seeded on top of the scaffold, the cells migrated through the complete scaffold volume in all groups. Constructs cultured at $300 \mathrm{rpm}$ showed more open structures, with rounded cells in between the scaffold, while statically cultured constructs and constructs cultured at $60 \mathrm{rpm}$ showed more elongated cells throughout the whole scaffold volume.

\section{Discussion}

The success of spinner flask bioreactors in BTE experiments is attributed to the creation of convective flow and the production of hydrodynamic forces that increase mass transport into the pores of the scaffold
(Griffon et al., 2011; Sikavitsas et al., 2002). Due to the complex flow generated within the bioreactor and the scaffold pores, the precise mechanical stimulation (i.e. WSS) acting on cells within a scaffold has not yet been investigated. The maximal mineralisation, based on WSS that correlates to the experimental observations is predicted. A CFD approach is employed to quantify the resultant mechanical stimulation (i.e. WSS) within an idealised scaffold, having a similar porosity and average pore size to the silk fibroin scaffold used in the current study. hMSCs were seeded on silk fibroin scaffolds and cultured in osteogenic medium in spinner flask bioreactors, either statically or dynamically. For the dynamic conditions, stirring rates of 60 or $300 \mathrm{rpm}$ were chosen so that differences in mineralised ECM formation, as predicted by the model, could be detected. A previous study indicates that WSS larger than $0.55 \mathrm{mPa}$ are able to induce the deposition of mineralised ECM within a silk fibroin scaffold (Vetsch et al., 2017). According 
to the model for the current study, only the bottom volume of the scaffold would be exposed to a WSS exceeding $0.55 \mathrm{mPa}$ at a stirring rate of $60 \mathrm{rpm}$. The experimental results confirmed that ECM production and mineralisation was, indeed, mainly at the bottom of the scaffold. For a stirring rate of $300 \mathrm{rpm}$, the computational model predicted that most of the scaffold volume should show mineralised ECM. This prediction was also supported by the experimental observation. Therefore, the location of mineralised ECM deposition within the silk fibroin scaffold could be associated directly with regions of high WSS, as predicted in the simulation, with a Pearson correlation coefficient of $97 \%$.

Without WSS simulations, no conclusion can be drawn concerning the effects of mechanical stimulation on cellular response. Previous studies, using spinner flask bioreactors for BTE, lack WSS simulations and probably are not intended to understand the effect of certain mechanical loads. Meinel et al. (2004) report the deposition of mineral within the peripheral region, at a stirring rate of $50 \mathrm{rpm}$. Goldstein et al. (2001) show increased calcium deposition at the scaffold's surface, using a spinner flask but without mentioning the stirring rate. Sucosky et al. (2004) introduce one of the first simulations, describing the flow field in a spinner flask bioreactor, without including the properties of the scaffold. A CFD approach is combined with experimental particle image velocimetry to assess the validity of the results. The prediction of velocitycomponents and maximum WSS level differ from the results by $20 \%$ and $16 \%$, respectively. Despite those differences between computational and experimental outcomes, the results are very encouraging as an initial tool to investigate the effects of culture medium convection. However, experimental verification using tissue engineered constructs is missing. Later, Bueno et al. (2005) perform simulations using a spinner flask and a wavy-walled bioreactor. Fluid flow inside the bioreactors is modelled using a turbulence model to generate velocity flow fields, according to Sucosky et al. (2004). The average WSS applied to constructs in the spinner flask is simulated to be twice as high as in the wavy-walled bioreactor, while showing enhanced axial mixing. The authors predict that the lower WSS should enhance aggregation of cartilaginous cells. After $28 \mathrm{~d}$ of culture, chondrocyte proliferation and matrix deposition are enhanced. Although the level of WSS is not related to the spatial distribution of ECM deposition in the scaffold, this study confirms the positive effects of the wavy-walled bioreactor for cartilage formation. To our knowledge, so far no one has simulated WSS in a scaffold within spinner flasks in order to predict cellular responses.

No mineralisation of the ECM was observed under static conditions. This was contradictory to other studies, which show mineralisation produced by hMSCs in osteogenic medium on silk fibroin scaffolds under static conditions (Hagenmüller et al., 2007; Sommer et al., 2016). Also, no GAGs and collagen were formed under static conditions. The lack of ECM formation and subsequent mineralisation, found in the current study, might have been due to variations in FBS used for cell culture or the cell donor. Differences in serum composition can lead to statistically significant differences of protein expression in human umbilical-vein endothelial cells (Bryan et al., 2011). There is also clear evidence for the influence of FBS types on mineralisation on silk fibroin scaffolds, both with and without cells seeded on them (Vetsch et al., 2015). Other FBS types or batches might show different mineralisation amounts and patterns on silk fibroin scaffolds. This is important to consider in future experiments and simulations. Models of bone development in vitro might have to be adjusted for each study, to account for such variations.

In addition to predicting the location of mineralised matrix, the computational model predicted that at $60 \mathrm{rpm}$ and $300 \mathrm{rpm}$, WSS at the upper and very top region, respectively, might be too low to induce mineralised ECM. Such low WSS, up to $0.39 \mathrm{mPa}$, are reported to induce proliferation in perfusion bioreactor experiments with similar silk fibroin scaffolds and hMSCs (Vetsch et al., 2017). In future studies, proliferation should be investigated in the different scaffold regions of constructs cultured at $60 \mathrm{rpm}$ and $300 \mathrm{rpm}$. For larger constructs, a spinner flask bioreactor would most likely provide an even more inhomogeneous WSS distribution (data not shown). Higher stirring rates would be necessary to ensure mechanical stimulation of cells in the upper scaffold volume. However, higher WSS would most likely reduce mineralisation in the scaffold bottom region due to too high mechanical stimulation, which might negatively affect the cells. McCoy et al. (2012), demonstrate that a mean WSS of $17.6 \mathrm{mPa}$ causes $20 \%$ of cells to detach from a collagen-GAG scaffold. Another study shows that WSS larger than $60 \mathrm{mPa}$ could result in cell death (Olivares et al., 2009). Correlating to the current CFD results, at a stirring rate of $300 \mathrm{rpm}$, the cells at the periphery of scaffold bottom region were likely to be detached or dead due to the high WSS (data not shown). However, ECM was deposited at the bottom of the scaffold, which implies that cells must have attached or migrated there during the culture period.

In dynamically cultured constructs, stirring might facilitate diffusion of extracellular matrix and signalling proteins from regions with greater osteogenic differentiation to regions with less osteogenic differentiation (Heino et al., 2004). However, facilitated diffusion of secreted extracellular matrix and signalling proteins probably played only a minor role in the experimental set up used in the current study. In constructs cultured at $60 \mathrm{rpm}$, secreted proteins from the lower osteogenically differentiated cells should have enhanced osteogenic differentiation in the upper part if diffusion was an influential parameter. In the $60 \mathrm{rpm}$ group localised osteogenic differentiation was only detected in the 
lower part of the scaffold, suggesting that either there was no convection of signalling proteins or that the secreted proteins were not able to induce sufficient osteogenic differentiation. In the $300 \mathrm{rpm}$ group all cells were mechanically stimulated which led to osteogenic differentiation throughout the whole construct, regardless of convection.

CFD is a useful tool to compute WSS in scaffold structures. A variety of simulation techniques are reported, from simple analytical to very complex computational models including, for example, $\mu \mathrm{CT}$ based scaffold structures (Goldstein et al., 2001; Jungreuthmayer et al., 2009; Maes et al., 2012). One of the limitations of the CFD model applied here is idealising and simplifying the scaffold geometry to save computational costs. The CFD model used in the current study was based on an idealised scaffold geometry, which had a similar porosity and average pore diameter to the silk fibroin scaffolds used in the study. Furthermore, four scaffolds were placed in the bioreactor, while the CFD model was based on only one scaffold. Even though an idealised scaffold geometry results in very low computational costs, compared to simulations of real scaffold geometries, it can cause a variation in the resultant WSS (Campos Marin and Lacroix, 2015). Such a simplification is likely to affect the flow velocity, which consequently might result in a variation of the WSS within the scaffolds. However, most scaffolds (proteins, polymers, gels etc.) when immersed in cell culture medium are not detectable using $\mu \mathrm{CT}$ scans and do not show comparable scaffold geometries in a dry and wet state. As a result, the real irregular scaffold geometry cannot be obtained for simulations and needs to be approximated (Porter et al., 2005; Zermatten et al., 2014). Scaffolds with a more regular scaffold geometry, manufactured using 3D printing, could facilitate computational simulation for BTE in the future (Egan et al., 2017). It should also be noted that the mechanical environment within the scaffold pores changes over time due to cell migration, cell proliferation and ECM deposition - this leads to dynamic changes in permeability. Therefore, knowing the scaffold structure at the beginning of the cell culture would only provide very limited information. While the initial cell seeding density is known to play an important role in bone tissue engineering (Vo et al., 2016; Xue et al., 2013), there are few reports of investigations into the relation between cell proliferation, migration and differentiation in 3D constructs. For example, Vetsch et al. (2017) show that low flow rates tend to induce cell proliferation and reduce osteogenic differentiation despite osteogenic medium being used, while high flow rates tend to induce osteogenesis. $\mu \mathrm{CT}$ imaging alone cannot not be used to visualise cell content and non-mineralised ECM growth longitudinally. Non-invasive monitoring techniques that follow tissue development over time would be invaluable to understanding the tissue regeneration process.

\section{Conclusion}

The current study showed that i) mechanical stimulation (i.e. WSS) could be generated and quantified within a porous scaffold in a spinner flask bioreactor; ii) mineralisation could be attributed to mechanical stimulation in a range of $0.55-10 \mathrm{mPa}$; iii) the model used could predict the location of mineralisation ECM and iv) hMSCs needed mechanical stimulation to differentiate along the osteogenic lineage and produce as well as mineralise ECM. CFD models could help determining optimal stimulation conditions for mineralisation in future bone TE experiments, to guide and understand bone development in vitro. More detailed models employing precise scaffold structures and taking tissue growth over time into account will be needed to understand which mechanical stimuli single cells experience in a 3D environment in vitro. Through the combination of CFD models with cell experiments, knowledge of the mechanical stimuli that cells experience can be linked to biological responses.

\section{Acknowledgements}

This project was supported by the European Union's Seventh Framework Programme (FP/2007-2013) / grant agreement No. 336043.

\section{References}

Alvarez-Barreto JF, Linehan SM, Shambaugh RL, Sikavitsas VI (2007) Flow perfusion improves seeding of tissue engineering scaffolds with different architectures. Ann Biomed Eng 35: 429-442.

Bryan N, Andrews KD, Loughran MJ, Rhodes NP, Hunt JA (2011) Elucidating the contribution of the elemental composition of fetal calf serum to antigenic expression of primary human umbilicalvein endothelial cells in vitro. Biosci Rep 31: 199-210.

Bueno EM, Bilgen B, Barabino GA (2005) Wavy-walled bioreactor supports increased cell proliferation and matrix deposition in engineered cartilage constructs. Tissue Eng 11: 1699-1709.

Campos Marin A, Lacroix D (2015) The intersample structural variability of regular tissueengineered scaffolds significantly affects the micromechanical local cell environment. Interface Focus 5: 20140097.

Egan PF, Gonella VC, Engensperger M, Ferguson SJ, Shea K (2017) Computationally designed lattices with tuned properties for tissue engineering using 3D printing. PLoS One 12: 1-20.

Gaspar DA, Gomide V, Monteiro FJ (2012) The role of perfusion bioreactors in bone tissue engineering. Biomatter 2: 167-175.

Gentile P, Chiono V, Carmagnola I, Hatton PV (2014) An overview of poly(lactic-co-glycolic) 
acid (PLGA)-based biomaterials for bone tissue engineering. Int J Mol Sci 15: 3640-59.

Goldstein AS, Juarez TM, Helmke CD, Gustin MC, Mikos AG (2001) Effect of convection on osteoblastic cell growth and function in biodegradable polymer foam scaffolds. Biomaterials 22: 1279-1288.

Griffon DJ, Abulencia JP, Ragetly GR, Fredericks LP, Chaieb S (2011) A comparative study of seeding techniques and three-dimensional matrices for mesenchymal cell attachment. J Tissue Eng Regen Med 5: 169-179.

Hagenmüller H, Hofmann S, Kohler T, Merkle HP, Kaplan DL, Vunjak-Novakovic G, Müller R, Meinel L (2007) Non-invasive time-lapsed monitoring and quantification of engineered bone-like tissue. Ann Biomed Eng 35: 1657-1667.

Heino TJ, Hentunen TA, Väänänen HK (2004) Conditioned medium from osteocytes stimulates the proliferation of bone marrow mesenchymal stem cells and their differentiation into osteoblasts. Exp Cell Res 294: 458-468.

Hildebrand T, Laib A, Muller R, Dequeker J, Ruegsegger P (1999) Direct three-dimensional morphometric analysis of human cancellous bone: microstructural data from spine, femur, iliac crest, and calcaneus. J Bone Miner Res 14: 1167-1174.

Hofmann S, Hagenmüller H, Koch AM, Müller R, Vunjak-Novakovic G, Kaplan DL, Merkle HP, Meinel L (2007) Control of in vitro tissue-engineered bonelike structures using human mesenchymal stem cells and porous silk scaffolds. Biomaterials 28: 1152-1162.

Hörmann T, Suzzi D, Khinast JG (2011) Mixing and dissolution processes of pharmaceutical bulk materials in stirred tanks: experimental and numerical investigations. Ind Eng Chem Res 50: 12011-12025.

Hulsart-Billstrom G, Dawson JI, Hofmann S, Muller R, Stoddart MJ, Alini M, Redl H, El Haj A, Brown R, Salih V, Hilborn J, Larsson S, Oreffo RO (2016) A surprisingly poor correlation between in vitro and in vivo testing of biomaterials for bone regeneration: results of a multicentre analysis. Eur Cell Mater 31: 312-322.

Jungreuthmayer C, Donahue SW, Jaasma MJ, Al-Munajjed AA, Zanghellini J, Kelly DJ, O'Brien FJ (2009) A comparative study of shear stresses in collagen-glycosaminoglycan and calcium phosphate scaffolds in bone tissue-engineering bioreactors. Tissue Eng Part A 15: 1141-1149.

Kapur S, Baylink DJ, Lau K-HW (2003) Fluid flow shear stress stimulates human osteoblast proliferation and differentiation through multiple interacting and competing signal transduction pathways. Bone 32: 241-251.

Karageorgiou V, Kaplan D (2005) Porosity of 3D biomaterial scaffolds and osteogenesis. Biomaterials 26: 5474-5491.

Kirkpatrick CJ, Fuchs S, Hermanns MI, Peters K, Unger RE (2007) Cell culture models of higher complexity in tissue engineering and regenerative medicine. Biomaterials 28: 5193-5198.
Klein-Nulend J, Bacabac RG, Bakker AD (2012) Mechanical loading and how it affects bone cells: the role of the osteocyte cytoskeleton in maintaining our skeleton. Eur Cell Mater 24: 278-291.

Lacroix D, Planell JA, Prendergast PJ (2009) Computer-aided design and finite-element modelling of biomaterial scaffolds for bone tissue engineering. Philos Trans A Math Phys Eng Sci 367: 1993-2009.

Lanyon LE (1993) Osteocytes, strain detection, bone modeling and remodeling. Calcif Tissue Int 53 Suppl 1: S102-107.

Maes F, Claessens T, Moesen M, Van Oosterwyck H, Van Ransbeeck P, Verdonck P (2012) Computational models for wall shear stress estimation in scaffolds: a comparative study of two complete geometries. J Biomech 45: 1586-1592.

Martin I, Wendt D, Heberer M (2004) The role of bioreactors in tissue engineering. Trends Biotechnol 22: 80-86.

McCoy RJ, Jungreuthmayer C, O'Brien FJ (2012) Influence of flow rate and scaffold pore size on cell behavior during mechanical stimulation in a flow perfusion bioreactor. Biotechnol Bioeng 109: 15831594.

Meinel L, Fajardo R, Hofmann S, Langer R, Chen J, Snyder B, Vunjak-Novakovic G, Kaplan D (2005) Silk implants for the healing of critical size bone defects. Bone 37: 688-698.

Meinel L, Karageorgiou V, Fajardo R, Snyder B, Shinde-Patil V, Zichner L, Kaplan D, Langer R, Vunjak-Novakovic G (2004) Bone tissue engineering using human mesenchymal stem cells: effects of scaffold material and medium flow. Ann Biomed Eng 32: 112-122.

Melke J, Midha S, Ghosh S, Ito K, Hofmann S (2016) Silk fibroin as biomaterial for bone tissue engineering. Acta Biomater 31: 1-16.

Menter FR (2009) Review of the shear-stress transport turbulence model experience from an industrial perspective. Int J Comut Fluid Dyn 23: 305-316.

Nazarov R, Jin H-J, Kaplan DL (2004) Porous 3-D scaffolds from regenerated silk fibroin. Biomacromolecules 5: 718-726.

Norvell SM, Alvarez M, Bidwell JP, Pavalko FM (2004) Fluid shear stress induces beta-catenin signaling in osteoblasts. Calcif Tissue Int 75: 396-404.

O’Keefe RJ, Mao J (2011) Bone tissue engineering and regeneration: from discovery to the clinic - an overview. Tissue Eng Part B Rev 17: 389-392.

Olivares AL, Marsal E, Planell JA, Lacroix D (2009) Finite element study of scaffold architecture design and culture conditions for tissue engineering. Biomaterials 30: 6142-6149.

Porter B, Zauel R, Stockman H, Guldberg R, Fyhrie D (2005) 3-D computational modeling of media flow through scaffolds in a perfusion bioreactor. J Biomech. 38: 543-549.

Sikavitsas VI, Bancroft GN, Mikos AG (2002) Formation of three-dimensional cell/polymer constructs for bone tissue engineering in a spinner 
flask and a rotating wall vessel bioreactor. J Biomed Mater Res 62: 136-148.

Sommer MR, Vetsch JR, Leemann J, Muller R, Studart AR, Hofmann S (2016) Silk fibroin scaffolds with inverse opal structure for bone tissue engineering. J Biomed Mater Res B Appl Biomater 105: 2074-2084.

Stiehler M, Bünger C, Baatrup A, Lind M, Kassem M, Mygind T (2009) Effect of dynamic 3-D culture on proliferation, distribution, and osteogenic differentiation of human mesenchymal stem cells. J Biomed Mater Res A 89: 96-107.

Sucosky P, Osorio DF, Brown JB, Neitzel GP (2004) Fluid mechanics of a spinner-flask bioreactor. Biotechnol Bioeng 85: 34-46.

Tortelli F, Pujic N, Liu Y, Laroche N, Vico L, Cancedda R (2009) Osteoblast and osteoclast differentiation in an in vitro three-dimensional model of bone. Tissue Eng Part A 15: 2373-2383.

Tsukada M, Gotoh Y, Nagura M, Minoura N, Kasai N, Freddi G (1994) Structural changes of silk fibroin membranes induced by immersion in methanol aqueous solutions. J Polym Sci Part B Polym Phys 32: $961-968$.

van Lenthe GH, Hagenmüller $H$, Bohner M, Hollister SJ, Meinel L, Müller R (2007) Nondestructive micro-computed tomography for biological imaging and quantification of scaffold-bone interaction in vivo. Biomaterials 28: 2479-2490.

Vetsch JR, Betts DC, Müller R, Hofmann S (2017) Flow velocity-driven differentiation of human mesenchymal stromal cells in silk fibroin scaffolds: A combined experimental and computational approach. PLoS One 12: e0180781.

Vetsch JR, Paulsen SJ, Müller R, Hofmann S (2015) Effect of fetal bovine serum on mineralization in silk fibroin scaffolds. Acta Biomater 13: 277-285.

Vo TN, Tabata Y, Mikos AG (2016) Effects of cellular parameters on the in vitro osteogenic potential of dual-gelling mesenchymal stem cell-laden hydrogels. J Biomater Sci Polym Ed 27: 1277-1290.

Volkmer E, Drosse I, Otto S, Stangelmayer A, Stengele M, Kallukalam BC, Mutschler W, Schieker M (2008) Hypoxia in static and dynamic 3D culture systems for tissue engineering of bone. Tissue Eng Part A 14: 1331-1340.

Woloszyk A, Dircksen SH, Bostanci N, Muöller R, Hofmann S, Mitsiadis TA (2014) Influence of the mechanical environment on the engineering of mineralised tissues using human dental pulp stem cells and silk fibroin scaffolds. PLoS One 9e0111010.

Xue R, Li JY-S, Yeh Y, Yang L, Chien S (2013) Effects of matrix elasticity and cell density on human mesenchymal stem cells differentiation. J Orthop Res 31: 1360-1365.

Yourek G, McCormick SM, Mao JJ, Reilly GC (2010) Shear stress induces osteogenic differentiation of human mesenchymal stem cells. Regen Med 5: 713-724.

Zermatten E, Vetsch JR, Ruffoni D, Hofmann S, Müller R, Steinfeld A (2014) Micro-computed tomography based computational fluid dynamics for the determination of shear stresses in scaffolds within a perfusion bioreactor. Ann Biomed Eng 42: 1085-1094.

Zhao F, Vaughan TJ, Mcnamara LM (2015) Multiscale fluid-structure interaction modelling to determine the mechanical stimulation of bone cells in a tissue engineered scaffold. Biomech Model Mechanobiol 14: 231-243.

\section{Discussion with reviewers}

Howard Winet: Do you expect flow velocity profiles over attached cells to be the same at both oscillatory Re values?

Authors' response: A steady state analysis was used in our CFD model, due to the constant rotational speed of the stirring bar in the experiment. Therefore, no oscillatory Re number is involved in our CFD model. According to Fig. 2, the flow velocity profile over the attached cells will not be the same at two different Re numbers. At a stirring rate of $300 \mathrm{rpm}$, fluid flowing from the scaffold top towards the bottom was more distinct due to vortex, as compared to $60 \mathrm{rpm}$. Thus, it was likely to influence the flow profile over the attached cells.

Yang Zhu: The simulation model generally matched the experimental results. However, other contributors need to be ruled out or analysed in order to strengthen the major conclusion of this study.

Authors' response: We agree with the reviewer that stimulated bone cells secrete paracrine factors which can regulate osteogenic differentiation and could diffuse throughout the scaffold by increased mass transport through stirring. Also, increased nutrient transport rather than mechanical stimulation might increase osteogenic differentiation. However, in constructs cultured at $60 \mathrm{rpm}$ localised osteogenic differentiation was detected in only the lower part of the scaffold, which suggested that either there was no convection of signalling proteins or that the secreted proteins were not able to induce sufficient osteogenic differentiation. In the $300 \mathrm{rpm}$ group all cells were mechanically stimulated which resulted in osteogenic differentiation throughout the whole construct, regardless of convection.

Editor's note: The Scientific Editor responsible for this paper was Stephen Ferguson. 